\title{
ON ASYMPTOTIC VALUES OF FUNCTIONS ANALYTIC IN A CIRCLE $\left({ }^{1}\right)$
}

\author{
BY \\ MAKOTO OHTSUKA
}

1. The class of functions $f(z)$, which are analytic and bounded, $|f(z)|<1$, in the unit circle $U:|z|<1$ and which have radial limit values of modulus 1 for almost all points $e^{i \theta}$ of $|z|=1$ is well known; for literature and general properties of these functions we refer the reader to the papers of W. Seidel [16] and A. J. Lohwater [10]. Some of the results mentioned in these papers can be obtained from general theorems in the theory of cluster sets of functions analytic in $U$ (cf. [4] and [13]). In recent papers Lohwater $[9 ; 10 ; 11]$ has extended the concept of this class to functions which are meromorphic in $U$ and whose moduli have radial limit 1 for almost all points of some arc $A$ of $|z|=1$. In particular, we cite the following result $[10 ; 11]:$ If $f(z)$ is meromorphic in $|z|<1$ with at most a finite number of zeros and poles and if $\lim _{r \rightarrow 1}\left|f\left(r e^{i \theta}\right)\right|=1$ for almost all $e^{i \theta}$ belonging to an $\operatorname{arc} A$ of $|z|=1$, then, unless $f(z)$ is analytic on $A$, there exists at least one curve (called an asymptotic path) terminating at a point of $A$ along which $f(z)$ tends either to 0 or $\infty$. If, in addition, $f(z)$ is of bounded characteristic in $|z|<1$, there exists at least one radius having this property.

In the present paper, we are motivated by Lohwater's results to define new boundary cluster sets of functions analytic in $U$ and taking values on an abstract Riemann surface $\Re$, and to establish relations between the cluster sets and the asymptotic values of the functions.

2. We begin with the definition of boundary points of an abstract Riemann surface $\Re$. Let $\mathfrak{F}$ be a class of filters such that each filter has a base consisting of open sets of $\Re$ which have no accumulation points on $\Re$. Furthermore we assume that of any two open sets of a base, one is contained in the other; that is, we have a nested base. We obtain a countable sub-base $\left\{G_{n}\right\}$ from the base if we take an exhaustion $\left\{\Re_{n}\right\}, \bar{\Re}_{n} \subset \Re_{n+1}$, with compact closures, and if we choose an element $G_{n}$ of the base so that $G_{n} \cap \Re_{n}=\varnothing$ for each $n$. For, given any element $G$ of the base, there is an $\Re_{n}$ such that $\Re_{n} \cap G \neq \varnothing$ and this shows $G_{n} \subset G$. Each filter of $\mathfrak{F}$ is defined to be $a$ boundary point of $\Re$, and we denote the set of all such boundary points by $\mathfrak{F}_{\Re}$. Let $P_{F}$ be a point of $\mathfrak{F}_{\Re}$ with a base $\left\{G_{n}\right\}$, and let $\left\{P_{\nu}\right\}$ be a sequence of points of $\Re+\mathfrak{F}_{\Re}$. If for each $n$ there exists an integer $\nu_{0}$ such that every $P_{\nu}, \nu \geqq \nu_{0}$, or some domain of its base, is contained in $G_{n}$, we say that $P_{\nu}$ converges to $P_{F}$. We keep the original definition of the convergence of points of $\Re$. Thus we obtain a topol-

Received by the editors October 13, 1953.

(1) This work was done at Harvard University under Contract N5ori-07634, NR043-046, with the Office of Naval Research. 
ogy for the space $\Re+\mathfrak{F}_{\Re}$. Boundary points obtained by the completion with respect to a metric in $\Re$ can be reinterpreted in the way above. The ramified boundary points and geodesic boundary points in [2] are examples.

3. Let $f(z)$ be an analytic function defined in $U:|z|<1$ and taking values on an abstract Riemann surface $\Re$ (with boundary $\mathfrak{F}_{\Re}$ if $\Re$ is open). For any set $E \subset U$ and any point $z_{0}$ on $C:|z|=1$ we define the cluster set $S_{z_{0}}^{(E)}$ at $z_{0}$ along $E$ to be the set of all values of $\Re+\mathfrak{F}_{\Re}$, for each point $P$ of which there exists a sequence of points $\left\{z_{n}\right\}$ of $E$ tending to $z_{0}$ such that $f\left(z_{n}\right) \rightarrow P$ as $\left.n \rightarrow \infty{ }^{2}\right)$. We shall write $S_{z_{0}}$ for $S_{z_{0}}^{(U)}$, and $T_{z_{0}}$ for the cluster set along the radius $O z_{0}$.

Let $\left\{K_{n}\right\}$ be an open base of $\Re$, and $z_{0}$ a point of $C$. If, for a given integer $n$, there exists at least one open $\operatorname{arc} C_{n}$ containing $z_{0}$ such that the inner linear measure of the set $\left\{z \in C_{n} ; z \neq z_{0}, T_{z} \cap K_{n} \neq \varnothing\right\}=C_{n}^{\prime}$ (which may be empty) is zero $\left({ }^{3}\right)$, we define $K_{n}^{*}$ by setting it equal to $K_{n}$; otherwise we put $K_{n}^{*}=\varnothing$. Denote the set $C-U_{\nu} C_{n_{\nu}}^{\prime}-z_{0}$ by $C^{*}$, where the summation $U_{\nu}$ is taken over all $n_{\nu}$ for which $K_{n_{\nu}}^{*}=K_{n_{\nu}}$. Next we take an open base $\left\{K_{\alpha}\right\}$ (this is not countable in general) of $\Re+\mathfrak{F}_{\Re}$ and define $K_{\alpha}^{*}$ in a similar way. We shall denote the set $\Re+\mathfrak{F}_{\Re}-\cup_{\alpha} K_{\alpha}^{*}$ by $S T_{z_{0}}$. This set is clearly a closed set in $\Re+\mathfrak{F}_{\Re}$ and may be considered as a sort of boundary cluster set $\left({ }^{4}\right)$.

Let us denote the intersection of any set $X$ with the circle $\left|z-z_{0}\right|<\rho$ by $X_{\rho}$. The cluster set $S T_{z_{0}}$ has a minimal property in the following sense: Taking any set $H \subset C, z_{0} \in H$, of linear measure zero, forming the closure $M_{\rho}^{(C-H)}$ of $\bigcup_{z \in(C-H)_{\rho}} T_{z}$, and denoting $\bigcap_{\rho}>0 M_{\rho}^{(C-H)}$ by $S T_{z_{0}}^{(C-H)}$, we have the relation $S T_{z_{0}} \subset S T_{z_{0}}^{(C-H)}$. The set $M_{\rho}^{\left(C^{*}\right)}$ will be used in the following Theorem 2.

If $f(z) \rightarrow P \in \Re+\mathfrak{F}_{\Re}$ along a curve in $U$ terminating at $z_{0}$, this curve is called an asymptotic path and the value $P$ an asymptotic value. The set of points on $\Re$ taken in any neighborhood in $U$ of $z_{0}$ is called the range of values and denoted by $R_{z_{0}}$.

4. We first prove the following lemma.

Lemma. Let $T$ be a continuous transformation of $U$ into a topological space $X$. Let $\Delta$ be a domain in $U$ whose image under $T$ is contained in a closed set $F$ in $X$ and, for almost all $e^{i \theta} \in \Delta^{b} \cap C$, where $\Delta^{b}$ is the boundary of $\Delta$, let the image of some end-part of the radius Oe $e^{i \theta}$ be contained in a closed set $F^{\prime}$, disjoint from $F$. If there exists a continuous real-valued function $g(P)$ in $X$ which assumes the value 0 on $F$ and 1 on $F^{\prime}$, then $m\left(\Delta^{b} \cap C\right)=0$.

Proof. We denote by $G(z)$ the function obtained by composing the transformation $T$ with $g(P)$. By our assumption $\lim _{r \rightarrow 1} G\left(r e^{i \theta}\right)=1$ at almost all points $e^{i \theta}$ of $\Delta^{b} \cap C$. By Egoroff's theorem, for any integer $p$ there exists a

(2) If $\Re+\mathfrak{F}_{\Re}$ is compact, $S_{z_{0}}^{(I)}$ is never empty whenever $z_{0}$ belongs to the closure of $E$.

(3) By means of the theory of functions of real variables, it can be proved that the set $C_{n}^{\prime}$ is linearly measurable. However, the set corresponding to $K_{\alpha}$ may be nonmeasurable in general.

(4) We used an idea in [4] in the definition of $S T_{z_{0}}$. 
closed subset $E_{p}$ of $\Delta^{b} \cap C$ such that $m\left(\Delta^{b} \cap C-E_{p}\right)<1 / p$ and $G\left(r e^{i \theta}\right)$ tends to 1 uniformly for $e^{i \theta} \in E_{p}$. Thus we can find $r_{1}<1$ such that $G(z)>1 / 2$ on the set $Y=\left\{r e^{i \theta} ; r_{1}<r<1, e^{i \theta} \in E_{p}\right\}$. We decompose the complement of $Y$ with respect to the annulus: $r_{1}<r<1$ into components $\left\{B_{n}\right\}(n=1,2, \cdots)$. Let $\left\{B_{n_{i}}\right\}$ be the components which have points in common with $\Delta$. Then its number is finite: $i=1,2, \cdots, k$. To prove this, suppose that there are an infinite number of $\left\{B_{n_{i}}\right\}$ having points in common with $\Delta$. Since $\Delta$ is a domain, we can connect a point of $B_{n_{1}} \cap \Delta$ with a point of each $B_{n_{i}} \cap \Delta(i \geqq 2)$ by a curve inside $\Delta$. This curve must cross the boundary arc of every $B_{n_{i}}$ on the circle: $|z|=r_{1}$. Any point of accumulation of these points of intersection is a boundary point of $\Delta$, and, at the same time, a point of $Y$. This is impossible because, by the continuity of $G(z), G(z)=0$ on the closure of $\Delta$ and $G(z)>1 / 2$ on $Y$. Therefore $\Delta^{b} \cap C$ is contained in $\left(U_{i-1}^{k} B_{n_{i}}\right)^{b} \cap C$. The linear measure of that part of $\Delta^{b} \cap C$ lying in the open intervals of $\left(\bigcup_{i=1}^{k} B_{n_{i}}\right)^{b} \cap C$ has the same value as $m\left(\Delta^{b} \cap C\right)$. But this part is the set $\Delta^{b} \cap C-E_{p}$ which has linear measure less than $1 / p$. Hence $m\left(\Delta^{b} \cap C\right)<1 / p$. Since $p$ is an arbitrary integer we see that $m\left(\Delta^{b} \cap C\right)=0$.

\section{Our theorems are}

THEOREM 1. Let $f(z)$ be an analytic function defined in $U$ and taking values on an abstract Riemann surface $\Re$ (with boundary $\mathfrak{F}_{\Re}$ if $\Re$ is open). Then a point $P_{0}$ of $S_{z_{0}}-S T_{z_{0}}-R_{z_{0}}$ is an asymptotic value at $z_{0}$ or at points $z_{n}$ of $C$ tending to $z_{0}$ if there exists a path in $\Re \cap S_{z_{0}}$ converging to $P_{0}$.

THEOREM 2. Let $f(z)$ be the same function as in Theorem 1. A point $P_{0}$ of the set $S T_{z_{0}}-R_{z_{0}}$ is an asymptotic value at $z_{0}$ or at points $z_{n}$ tending to $z_{0}$ if

(i) there exists a number $\rho>0$ such that there is a path in $\Re \cap\left(S_{z_{0}}-M_{\rho}^{\left(C^{*}\right)}\right)$ converging to $P_{0}$, and if

(ii) the set of points on $|z|=1$ where the radial cluster sets $T_{z}$ do not contain $P_{0}$ is everywhere dense in a certain open arc $C^{\prime} \subset C$ containing $z_{0}$.

We shall prove Theorem 2 for $P \in F_{\Re}$. The proof for the case $P \in \Re$ and the proof of Theorem 1 are easily obtained by modifying the proof given below.

Let $L$ be the path in $\Re \cap\left(S_{z_{0}}-M_{\rho}^{\left(C^{*}\right)}\right)$, converging to $P_{0}$. We form two paths on each side of $L$ and close enough to $L$ that the domain $D$ between them is contained in $\Re-M_{\rho}^{\left(C^{*}\right)}$. Let $\left\{G_{n}\right\}$ be a nested countable base of the filter defining $P_{0}$ and let $D_{n}$ be that component of the intersection of $G_{n}$ with $D$ which contains an end-part of $L$. Obviously, $D_{1} \supset D_{2} \supset \cdots \rightarrow P_{0}$.

We take two points $z_{1}$ and $z_{2}$ on $C$ near $z_{0}$ so that $\arg z_{1}<\arg z_{0}<\arg z_{2}$, $\left|z_{0}-z_{1}\right|<\rho$ and $\left|z_{0}-z_{2}\right|<\rho$, and such that $T_{z_{1}} \cup T_{z_{2}}$ does not contain $P_{0}$. Let $r^{\prime}<1$ be a number sufficiently near 1 . Denote the sector $\left\{r e^{i \theta} ; r^{\prime}<r<1\right.$, $\left.\arg z_{1}<\theta<\arg z_{2}\right\}$ by $Q$ and its boundary inside $U$ by $q$. We may assume that the image of $q$ lies outside some neighborhood of $P_{0}$. The inverse image of $D_{n}$ in $Q$ is not empty since $L \subset S_{z_{0}}$. For $n$ sufficiently large, some component, 
say $\Delta_{n}$, together with its closure, has no common point with $q$.

Suppose that, in $\Delta_{n}, f(z)$ does not assume values of $D_{n+1}$. Then the closure of the image $f\left(\Delta_{n}\right)$ of $\Delta_{n}$ is compact in $\Re$, and for almost all $z$ of $C_{p}$ the radial cluster sets $T_{z}$ lie outside the closure of $f\left(\Delta_{n}\right)$. Then by our lemma, the measure $m\left(\Delta_{n}^{b} \cap C\right)=0$, the continuous function $g(P)$ of the lemma being defined by the aid of a metric in $\Re$. Therefore the harmonic measure of $\Delta_{n}^{b} \cap C$ with respect to $\Delta_{n}$ is zero. We take a small compact Jordan domain $K_{0}$ inside $D_{n+1}$ and form a harmonic measure function of the boundary of $K_{0}$ in the domain $D_{n}-K_{0}$. If we regard this function as a function defined in $\Delta_{n}$, it has boundary value 0 except for points of $\Delta_{n}^{b} \cap C$ which has harmonic measure zero. By the maximum principle this function must be the constant zero, which is a contradiction.

Thus we have shown that $f\left(\Delta_{n}\right) \cap D_{n+1} \neq \varnothing$. Consider the inverse image of $D_{n+1}$ in $\Delta_{n}$ and let $\Delta_{n+1}$ be any component of the image. We can show as above that $f\left(\Delta_{n+1}\right) \cap D_{n+2} \neq \varnothing$. In this manner we obtain a sequence of domains $\Delta_{n} \supset \Delta_{n+1} \supset \cdots$ where $f\left(\Delta_{k}\right) \subset D_{k}(k=n, n+1, \cdots)$. Taking a point $z_{k}$ in $\Delta_{k}$ and connecting it with any point $z_{k+1}$ of $\Delta_{k+1}$ by a curve in $\Delta_{k}$, we get a path $l$ in $Q$ along which $f(z) \rightarrow P_{0}$. By assumption (ii) (we may suppose that the arc $z_{1} z_{2}$ is contained in $\left.C^{\prime}\right), l$ terminates at a single point of $|z|=1$. Since $Q$ may be taken arbitrarily near $z_{0}$ the conclusion of Theorem 2 is obtained.

REMARK. If we allow a path to oscillate, we may infer the existence of such a path with asymptotic value $P_{0}$ in any neighborhood of $z_{0}$, with the following condition replacing (ii):

(ii') there exist points $\zeta$ on $|z|=1$ on both sides of $z_{0}$ and arbitrarily close to $z_{0}$ such that $P_{0}$ does not belong to $T_{\xi}$. If we assume only (i), then we know that either there is a path (which may oscillate) in any neighborhood of $z_{0}$, or there is a sequence of curves which accumulate on a closed arc containing $z_{0}$, such that $f(z) \rightarrow P_{0}$ uniformly along these curves $\left({ }^{5}\right)$.

6. In the theory of cluster sets the difference between a cluster set such as $S_{z_{0}}$ and a boundary cluster set such as $S T_{z_{0}}$ is an open set. In the case of an abstract Riemann surface, it is not generally true that $S_{z_{0}}-S T_{z_{0}}$ is an open set in $\Re+\mathfrak{F}_{\Re}$. On the one hand, suppose that there is a point $P_{0} \in \Re \cap\left(\left(S_{z_{0}}\right)^{b}\right.$ $\left.-S T_{z_{0}}\right)$. Since $S T_{z_{0}}$ is a closed set, there is a domain $G$ on $\Re$, containing $P_{0}$ and with compact closure corresponding to a parametric circle $|\omega| \leqq 1$, such that $\bar{G} \cap S T_{z_{0}}=\varnothing$. We denote the open set which is the inverse image in $U$ of $G$ by $\Delta$, and its boundary by $\delta$. It follows from the lemma that, if we take a part $H$ of $\delta \cap C$ sufficiently near $z_{0}$, its linear measure, and hence its relative harmonic measure with respect to $\Delta$, is zero. The cluster set $S_{z_{0}}^{(\Delta)}$ of the composed function $\omega(f(z))$ contains the point $\omega=0$ but does not coincide with the whole $|\omega| \leqq 1$, and the boundary cluster set $S_{z_{0}}^{(\delta-H)}$ (this is defined by setting

(5) This fact is expressed by the notation $P_{0} \in \Phi(f)$ in [6]. 
$E=\delta-H$ in $S_{z_{0}}^{(L)}$ of $\S 3$ ) is contained in $|\omega|=1$. This fact contradicts the following theorem which is easily deduced from a theorem in M. Brelot [1]: Let $\Delta$ be an open set with boundary $\delta$ in the $z$-plane, $z_{0}$ a nonisolated boundary point of $\Delta, H$ a subset of $\delta$, containing $z_{0}$, of relative harmonic measure zero with respect to $\Delta$, and $f(z)$ analytic in $\Delta$ and on $\delta-H$. Then the difference between the cluster set $S_{z_{0}}^{(\Delta)}$ and the boundary cluster set $S_{z_{0}}^{(\delta-H)}$ is an open set.

We state our result in

THEOREM 3(6). Under the assumption of Theorem $1, \Re \cap\left(S_{z_{0}}-S T_{z_{0}}\right)$ is an open set.

On the other hand, however, we shall show that $S_{z_{0}}-S T_{z_{0}}$ is not necessarily open. Let $\Re$ be the circle $|w|<1$ and suppose that $\mathfrak{F}_{\Re}$ consists of only one point $w_{0}$ on $|w|=1$. Let $f(z)$ be the identity function: $w=z\left(w_{0}=z_{0}\right)$. Then $S_{z_{0}}=\left\{w_{0}\right\}$ but $S T_{z_{0}}=\varnothing$, so that $S_{z_{0}}-S T_{z_{0}}=\left\{w_{0}\right\}$ is a closed set.

7. We shall discuss next some special cases of Theorems 1 and 2. Suppose first that $f(z)$ possesses a radial limit almost everywhere on an open $\operatorname{arc} A$ of $|z|=1$ containing $z_{0}$. Then $S T_{z_{0}}$ is the intersection of the closures of certain sets of such limit values. For instance, if the range of values $R_{z_{0}}$ is compact relatively in $\Re$ and does not cover a set of positive logarithmic capacity, $f(z)$ has this property $\left({ }^{7}\right)$.

We have next the following corollary to Theorem 1:

COROLlaRy $1\left(^{8}\right)$. Let $R_{z_{0}}$ be conformally equivalent neither to the Riemann sphere punctured at most two points nor to a torus. Then any value $P_{0}$ of $\Re$ belonging to $S_{z_{0}}-S T_{z_{0}}-R_{z_{0}}$ is a radial limit either at $z_{0}$ or at points $z_{n}$ tending to $z_{0}$.

First we observe that there is a path in $\Re \cap S_{z_{0}}$ terminating at $P_{0}$ since a neighborhood of $P_{0}$ is contained in $S_{z_{0}}$ by Theorem 3. By Theorem 1 we can then obtain a path terminating at a point $z^{\prime}$ of $C$ near $z_{0}$, with the asymptotic value $P_{0}$. We can prove by the generalization of Lindelöf's theorem that $f(z)$ has the same asymptotic value $P_{0}$ along the radius with the end point $z^{\prime}$.

We consider the class of functions studied by Lohwater [11]: A function $f(z)$, meromorphic in $|z|<1$, is said to belong to class $\left(U^{*}\right)$ if there exists an arc $A$ of $C$ such that $\lim _{r \rightarrow 1}\left|f\left(r e^{i \theta}\right)\right|=1$ for almost all $e^{i \theta}$ of $A$.

Corollary $2[10 ; 11]$. Let $w=f(z)$ be a function of class $\left(U^{*}\right)$. If $f(z)$ is not analytic on the arc $A$ and if it possesses at most a finite number of zeros and poles in a neighborhood of $A$, then there exists at least one curve, terminating at a point of $A$, along which $f(z) \rightarrow 0$ or $\infty$.

From an extension of Schwarz's symmetry principle [4] it follows that, at any singular point $z_{0}$ of $A$, at least one of 0 and $\infty$ belongs to $S_{z_{0}}$. Since

$\left(^{8}\right)$ This was proved in [4] and [13] in the case when $\Re$ is the extended $w$-plane.

( 7 ) See Theorem 3.3 in [14].

(8) The case when $R$ is the whole plane and $S_{\varepsilon_{0}}$ is not was discussed in [13]. 
$S T_{z_{0}} \subset\{|w|=1\}$, and since $S_{z_{0}}-S T_{z_{0}}$ is an open set by Theorem 3 , the assumptions of Theorem 1 are satisfied for $P_{0}=0$ or $\infty$; hence Corollary 2 is established.

In connection with Theorem 2 , we remark that if $R_{z_{0}}$ satisfies the conditions of Corollary 1 , it cannot happen that $f(z)$ tends to a value in $\Re$ uniformly on a sequence of curves accumulating on an arc of $C$ near $z_{0}$. Therefore for such a function condition (ii) in Theorem 2 is not necessary if the point $P_{0}$ belongs to $\Re$. However, we do not know whether condition (ii) is necessary in general, even for the functions of class $\left(U^{*}\right)\left({ }^{9}\right)$, although condition (i) is fulfilled for these functions.

We shall prove

Corollary 3 (Calderón-Domingues-Zygmund [3], cf. also [8]). Let $w=f(z)$ be a bounded analytic function defined in $|z|<1$. Let $f(z)$ have a radial limit of modulus one almost everywhere on an arc $A$ of $C$. Then if $f(z)$ is not analytic on $A$, every value of $|w|=1$ is a radial limit at infinitely many points of $A$.

Let $z_{0} \in A$ be a singular point. By Theorem 2 and Lindelöf's theorem any point $w$ of $|w|=1$ is the radial limit at $z_{0}$ or at $z_{n}$ tending to $z_{0}$. If such $\left\{z_{n}\right\}$ exists, the corollary is already proved. Also if there are singular points on $C$ tending to $z_{0}$, then our corollary follows. Hence suppose that $f(z)$ were analytic on $C$ near $z_{0}$, except at $z_{0}$, and $f(z) \neq w$ there. In this situation $f(z)$ would have limit values $w_{1}$ and $w_{2}$ respectively as $z \in C$ moves toward $z_{0}$ from both sides. Since $f(z)$ is bounded, $w_{1}=w_{2}$ and $f(z)$ would tend to this value uniformly as $z$ approaches $z_{0}$ from the inside of $U$ by Lindelöf's theorem. Then $f(z)$ would be analytic at $z_{0}$ and this is a contradiction. Thus the corollary is proved.

8. In this section we shall consider mermorphic functions of bounded characteristic. Such a function has radial limit almost everywhere, and the set of points of $C$ where the function has the same radial limit has linear measure zero by Riesz-Nevanlinna's theorem. Therefore condition (ii) of Theorem 2 is not necessary. However, we can show by an example that at a point where an asymptotic path terminates, the function does not always have a radial limit of the same value. We know that this is true for a meromorphic function with at least three exceptional values. Hence let us remark that the following theorem is not a special case of Corollary 1 if and only if $f(z)$ omits only two values.

THEOREM 4. Let $w=f(z)$ be a meromorphic function of bounded characteristic in $U$, and suppose that it does not take two values $w_{0}$ and $w_{1}$ near a point $z_{0}$ of $|z|=1$. If $w_{0}$ belongs to $S_{z_{0}}-S T_{z_{0}}$, then $w_{0}$ is a radial limit at $z_{0}$ or at points $z_{n}$ tending to $z_{0}$.

(9) In a recent letter Professor Lohwater remarked to the author that his unpublished proof of the first part of [9] is not complete and that that aspect of the question is still open. 
Proof $\left({ }^{10}\right)$. Without loss of generality we may suppose that $w_{0}=0, w_{1}=\infty$. and $S T_{z_{0}}$ lies outside $|w| \leqq 1$. Then $f(z)$ has a representation (see [12])

$$
f(z)=\frac{\Omega_{1}(z)}{\Omega_{2}(z)} \exp \left[\frac{1}{2 \pi} \int_{0}^{2 \pi} \frac{e^{i \phi}+z}{e^{i \phi}-z} d \mu(\phi)+i \lambda\right],
$$

where $\Omega_{1}(z)$ and $\Omega_{2}(z)$ are finite Blaschke products, $\lambda$ is a real constant, and $\mu(\theta)$ is a function of bounded variation in $[0,2 \pi]$ such that $\mu(\theta)=\{\mu(\theta+)$ $+\mu(\theta-)\} / 2$. In order to prove our theorem it is sufficient to prove that

$$
u(z)=\frac{1}{2 \pi} \int_{0}^{2 \pi} \frac{1-r^{2}}{1+r^{2}-2 r \cos (\phi-\theta)} d \mu(\phi) \quad\left(z=r e^{i \theta}\right)
$$

tends to $-\infty$ along a radius at $z_{0}$ or at a point arbitrarily near $z_{0}$.

Let $C_{\rho}$ be an arc such that the set $M_{\rho}^{\left(C^{*}\right)}$ defined in $\$ 3$ lies outside $|w| \leqq 1$, and let us decompose $u(z)$ into the integral $u_{1}(z)$ on $C_{\rho}$ and the integral $u_{2}(z)$ on its complement.

We denote the set function corresponding to $\mu(\theta)$ by $\mu^{*}(X)$. Then by de la Vallée Poussin's decomposition theorem (see $[15$, p. 127]) we have

$$
\mu^{*}(X)=\mu^{*}\left(X \cap E_{+\infty}\right)+\mu^{*}\left(X \cap E_{-\infty}\right)+\int_{X} \mu^{\prime}(\phi) d(\phi)
$$

for any Borel set $X$ consisting of points of continuity of $\mu(\theta)$, where $E_{+\infty}$ and $E_{-\infty}$ represent the sets of points at which $\mu(\theta)$ has derivatives equal to $+\infty$ and $-\infty$ respectively. According to Fatou's theorem, $u\left(r e^{i \theta}\right) \rightarrow \mu^{\prime}(\theta)$ as $r \rightarrow 1$ for almost all $\theta$. By hypothesis, $\left|f\left(r e^{i \theta}\right)\right|=\exp \left[u\left(r e^{i \theta}\right)\right]$ tends to $\exp \left[\mu^{\prime}(\theta)\right]>1$ as $r \rightarrow 1$ for almost all $e^{i \theta}$ of $C_{\rho}$. Hence $\mu^{\prime}(\theta)>0$ for almost all $e^{i \theta}$ of $C_{\rho}$. Now it is a result of Lohwater [10, Lemma] that if there is a negative jump of $\mu(\theta)$ on $C, u(z)$ tends to $-\infty$ radially at this point. We now suppose that $\mu(\theta)$ has no negative jump on $C_{\rho}$. Let $Y$ be any Borel subset of $C_{\rho}$ which does not contain points of discontinuity of $\mu(\theta)$. If $E_{-\infty} \cap C_{\rho}=\varnothing$, then the positiveness of $\mu^{*}(Y)$ follows from the above equality because $\mu^{*}\left(Y \cap E_{+\infty}\right)$ is always non-negative (see lemma in $\left[15\right.$, p. 126]). Let $X$ be any Borel subset of $C_{\rho}$, and $\left\{a_{n}\right\}$ be the points of discontinuity of $\mu(\theta)$ on $X ;\left\{a_{n}\right\}$ coincide with the jumps of $\mu(\theta)$. Then $\mu^{*}(X)=\mu^{*}\left(X-\left\{a_{n}\right\}\right)+\sum_{n} \mu^{*}\left(a_{n}\right)$ and $\mu^{*}\left(a_{n}\right)$ is equal to the saltus at $a_{n}$. Since both terms of the right side are positive, $\mu^{*}(X)>0$. Therefore $u_{1}(z)>0$ and hence $u(z)>m>-\infty$ near $z_{0}$. Hence $|f(z)|>m_{1}>0$ near $z_{0}$. This contradicts the assumption that $0 \in S_{z_{0}}$. Thus there is at least one point $e^{i \theta}$ of $E_{-\infty}$ on $C_{\rho}$. At this point $u(z)$ has a radial limit $-\infty$. On account of the arbitrariness of $C_{\rho}$, the theorem is concluded.

As mentioned in $\$ 1$, a special case of this theorem was proved in [10]. Whether the existence of $w_{1} \in R_{z_{0}}$ in our theorem is necessary or not is not yet determined. Also notice that we have no such result corresponding to Theo-

(10) The writer owes the idea of the proof to [10]. 
rem 2.

9. In order to determine more completely the asymptotic values of an analytic function, we shall define a smaller boundary cluster set. Up to now we have used the radial cluster set $T_{z_{0}}$ to define the boundary cluster set $S T_{z_{0}}$. In many cases the set $T_{z_{0}}$ is too large; we shall find it more suitable to use, instead, the set $\Gamma_{z_{0}}$ of all asymptotic values at $z_{0}$, in some cases.

Let $f(z),\left\{K_{n}\right\},\left\{K_{\alpha}\right\}$ and $z_{0}$ be the same as in $\S 3$. We shall use the same notation as in $\$ 3$, whenever it causes no confusion. If, for a given integer $n$, there is an open $\operatorname{arc} C_{n}$ containing $z_{0}$ such that the inner linear measure of the set $\left\{z \in C_{n} ; z \neq z_{0}, \Gamma_{z} \cap K_{n} \neq \varnothing\right\}=C_{n}^{\prime}$ is zero, we define $K_{n}^{*}$ by $K_{n}$; otherwise we set $K_{n}^{*}=\varnothing$. We denote the set $C-U_{\nu} C_{n_{p}}^{\prime}-z_{0}$ by $C^{*}$, where the summation $\bigcup_{\nu}$ is taken over all $n_{\nu}$ for which $K_{n_{\nu}}^{*}=K_{n_{\nu}}$. We define $K_{\alpha}^{*}$ in a similar fashion for $\left\{K_{\alpha}\right\}$ and set $S \Gamma_{z_{0}}=\Re+\Im_{\Re}-\bigcup_{\alpha} K_{\alpha}^{*}$. This set $S \Gamma_{z_{0}}$ is the smallest set in the following sense: Let $H \subset C, z_{0} \in H$, be a set of linear measure zero, and denote the closure of $\bigcup_{z \in(C-H)_{\rho}}^{\prime} \Gamma_{z}$ by $N_{\rho}^{(C-H)}$ and the intersection $\bigcap_{p>0} N_{\rho}^{(C-H)}$ by $S \Gamma_{z_{1}}^{(C-H)}$. Then $S \Gamma_{z_{0}} \subset S \Gamma_{z_{1}}^{(C-H)}$.

The following theorems correspond respectively to Theorems 1 and 2:

THEOREM 5. Let $f(z)$ be an analytic function defined in $|z|<1$ and taking values of an abstract Riemann surface $\Re$ (with boundary $\mathfrak{F}_{\Re}$ if $\Re$ is open). Then a point $P_{0}$ of $S_{z_{0}}-S \Gamma_{z_{0}}-R_{z_{0}}$ is an asymptotic value either at $z_{0}$ or at points $z_{n}$ tending to $z_{0}$ if (i) there exists a path in $\Re \cap S_{z_{0}}$ converging to $P_{0}$, and if (ii) there exists a set $E$ of points on $|z|=1$, dense in some neighborhood of $z_{0}$, such that, for each $\zeta \in E$, there is a path $l_{\zeta}$ on $|z|<1$ terminating at $\zeta$ with the property that the cluster set of $f(z)$ along $l_{5}$ does not contain $P_{0}$.

Theorem 6. Let $f(z)$ be the same as in Theorem 5. A point $P_{0}$ of $S \Gamma_{z_{0}}-R_{z_{0}}$ is an asymptotic value either at $z_{0}$ or at points $z_{n}$ tending to $z_{0}$ if (i) there exists a path in $\Re \cap\left(S_{z_{0}}-N_{\rho}^{\left(C^{*}\right)}\right)$ converging to $P_{0}$, where $\rho$ is a certain positive number, and if condition (ii) of Theorem 5 is satisfied.

Notice that condition (ii) is required even in Theorem 5. If we lift this requirement in Theorems 5 and 6 , then the same remark as in $\$ 5$ is given.

The proofs for these theorems are similar to but simpler than those for Theorems 1 and 2 and are omitted here. We shall explain Theorem 5 in a special case.

Let $w=f(z)$ be a meromorphic function defined in $|z|<1$ and suppose that $f(z)$ omits at least three values in a certain neighborhood of a point $z_{0}$ on $|z|$ $=1$. Then the points of $|z|=1$ which have radial limits are everywhere dense in an open arc containing $z_{0}$ (see [5] and [13]). Therefore condition (ii) of Theorem 5 is not necessary. If $S_{z_{0}}$ is the whole $w$-plane, condition (i) in Theorem 5 is clearly fulfilled, while, if $S_{z_{0}}$ is not the whole $w$-plane, Theorem 5 is contained in Theorem 1. Therefore, for our function $f(z)$, Theorem 5 may be stated without conditions (i) and (ii) (this theorem was stated in [13]). The 
modular function shows that Theorem 5 is not contained in Theorem 1.

We remark that $\Re \cap\left(S_{z_{0}}-S \Gamma_{z_{0}}\right)$ is an open set. This is trivial if $\Re \subset S_{z_{0}}$, and if $\Re \nsubseteq S_{z_{0}}$ then $\Re \cap S \Gamma_{z_{0}}=\Re \cap S T_{z_{0}}\left({ }^{11}\right)$ and our assertion follows from Theorem 3.

10. Finally we shall examine the assumptions of Theorems 1 and 2. Let $\Re$ be a simply-connected domain in the $w$-plane which spirals down on $|w|=1$ from the outside and suppose that $\mathfrak{F}_{\Re}$ consists of only one point $w_{0}$ of $|w|=1$ with the ordinary topology. We map $\Re$ onto $|z|<1$ and denote by $z_{0}$ the point on $|z|=1$ which corresponds to $w_{0}$. Then $S_{z_{0}}=\left\{w_{0}\right\}$ and $S T_{z_{0}}=\varnothing$. Clearly $w_{0}$ is never an asymptotic value; here, the condition in Theorem 1 is not satisfied. However, if we take $P_{0} \in S_{z_{0}}-S T_{z_{0}}$ in $\Re$, then the required curve is obtained by Theorem 3 .

The following examples show that condition (i) is necessary in Theorem 2 even if we take the point $P_{0}$ in $\Re$.

EXAmple 1. Take the circles $U_{w}:|w|<1$ and $V_{n}:|w-1| \leqq 1 / n$ $(n=1,2, \cdots)$. Set $U_{w}-V_{n}=G_{n}$ and connect $G_{n}$ and $G_{n+1}$ by a small strip domain $S_{n}$ near the point $w=-1$ so that $S_{n} \rightarrow-1$ as $n \rightarrow \infty\left({ }^{12}\right)$ and $G_{1} \cup S_{1}$ $\cup G_{2} \cup S_{2} \cup \ldots$ is a simply-connected Riemann surface $\Re_{1}$. Map $\Re_{1}$ onto $U:|z|<1$ conformally. Then by Koebe's theorem the image of $S_{n}$ and hence the image of $G_{n}$ tends to a point, say $z_{0}$, of $C:|z|=1$, and $z_{0}$ is the only point which is not an image of any boundary point of $\left\{G_{n}\right\}$ and $\left\{S_{n}\right\}$. For the function $w=f(z)$ mapping $U$ into the w-plane through $\Re_{1}$, the cluster sets are $S_{z_{0}}=\{|w| \leqq 1\}$ and $S T_{z_{0}}=S \Gamma_{z_{0}}=\{|w|=1\}$. The point $w=1$ is neither taken by $f(z)$ nor is an asymptotic value; the path which is required in (i) of Theorem 2 actually does not exist.

EXAMPLE $2\left({ }^{13}\right)$. We shall construct a similar example in which $w=1$ is the only one exceptional value (i.e. $R_{z_{0}}=\{w \neq 1\}$ ). Let $\left\{V_{n}\right\}$ be the same as in the first example. Let us set $A_{n}=\{|w|<1+1 / n\}-V_{n}$ and $B_{n}=\{|w|>1$ $-1 / n\}-V_{n}$, and let us connect $A_{n}$ with $B_{n}$ by a strip $S_{n}$ and $B_{n}$ with $A_{n+1}$ by a strip $S_{n}^{\prime}$ so that these strips $S_{n}$ and $S_{n}^{\prime}$ tend to $w=-1$ as $n \rightarrow \infty$ and $A_{1} \cup S_{1} \cup B_{1} \cup S_{1}^{\prime} \cup A_{2} \cup S_{2} \cup B_{2} \ldots$ is a simply-connected Riemann surface $\Re_{1}$. We map $\Re_{1}$ onto $U$ conformally and denote the function corresponding to the mappings $U \rightarrow \Re_{1} \rightarrow$ the $w$-plane by $f(z)$. We shall construct $\left\{S_{n}\right\}$ so that the $z$-images of $A_{n}, B_{n}, S_{n}$, and $S_{n}^{\prime}$ tend to a point of $C$ as $n \rightarrow \infty$.

Suppose that each $S_{n}$ contains a part $S_{n}^{*}$ which is mapped conformally onto a rectangle: $0<u<a_{n}, 0<v<b_{n}$ such that the sides with length $a_{n}$ correspond to a part of the boundary of $\Re_{1}$. Consider, on $S_{n}^{*}$, the function which maps $\Re_{1}$ onto $U$, we transform it into the function defined on the rectangle and denote it by $z=g(u+i v)$. This is a schlicht function, and we have, by Schwarz's inequality,

(11) We can prove this as for Theorem 3.3 of [14].

(12) For instance, take the part of $1 / n+1<|w+1|<1 / n$ outside $U_{w}$ as $S_{n}$.

(13) The writer owes some technique in the construction of this example to [7]. 


$$
\begin{aligned}
\left\{\int_{0}^{a_{n}} \int_{0}^{b_{n}}\left|g^{\prime}(u+i v)\right| d u d v\right\}^{2} & \\
& \leqq\left\{\int_{0}^{a_{n}} \int_{0}^{b_{n}}\left|g^{\prime}(u+i v)\right|^{2} d u d v\right\}\left\{\int_{0}^{a_{n}} \int_{0}^{b_{n}} d u d v\right\}
\end{aligned}
$$

If the images of $A_{n}, S_{n}, B_{n}$, and $S_{n}^{\prime}$ do not tend to a point, they must tend to some arc, say $z_{1} z_{2}$. Then, denoting the area of the image of $S_{n}$ by $S_{n}$, we get from the above inequality

$$
\left(\left|z_{1}-z_{2}\right| \cdot a_{n}\right)^{2} \leqq s_{n} \cdot a_{n} b_{n}
$$

whence

$$
0<\left|z_{1}-z_{2}\right|^{2} \leqq s_{n} b_{n} / a_{n}
$$

Since $s_{n} \rightarrow 0$ as $n \rightarrow \infty$, there arises a contradiction if we assume $b_{n} / a_{n}<M<\infty$. Therefore under the assumption that $S_{n}$ contains such a part $S_{n}^{*}$ (this means that $S_{n}^{*}$ is "narrow") it is proved that $A_{n}, S_{n}, B_{n}$, and $S_{n}^{\prime}$ tend to a point, say $z_{0}$, of $C$. In this example $w=1$ is the only one exceptional value and $S T_{z_{0}}=S \Gamma_{z_{0}}$ coincides with $|w|=1$. The point $w=1$ is not an asymptotic value; actually condition (i) in Theorem 2 is not fulfilled(14).

Whether condition (ii) in Theorem 2 is really necessary or not is not yet known, as already stated in $\$ 7$.

\section{BIBLIOGRAPHY}

1. M. Brelot, Sur l'allure d la frontière des fonctions harmoniques, sousharmoniques ou holomorphes, Bulletin de la Société Royale des Sciences de Liège (1939) pp. 468-477.

2. M. Brelot and G. Choquet, Espaces et lignes de Green, Ann. L'Inst. Fourier vol. 3 (1952) pp. 199-263.

3. A. P. Calderón, A. Gonzáles-Domingues, and A. Zygmund, Note on the limit values of analytic functions, Revista de la Unión Matemática Argentina vol. 14 (1949) pp. 16-19 (Spanish).

4. C. Carathéodory, Zum Schwarzschen Spiegelungsprinzip, Comment. Math. Helv. vol. 19 (1946-47) pp. 263-278.

5. M. L. Cartwright, On the behaviour of an analytic function in the neighbourhood of its essential singularities, Math. Ann. vol. 112 (1936) pp. 161-187.

6. E. F. Collingwood and M. L. Cartwright, Boundary theorems for a function meromorphic in the unit circle, Acta Math. vol. 87 (1952) pp. 83-146.

7. W. Gross, Über die Singularitäten analytischer Funktionen, Monatshefte für Mathematik Physik vol. 29 (1918) pp. 3-47.

8. M. Heins, Studies in the conformal mapping of Riemann surfaces, I, Proc. Nat. Acad. Sci. U.S.A. vol. 39 (1953) pp. 322-324. p. 470.

9. A. J. Lohwater, On the Schwarz reflexion principle, Bull. Amer. Math. Soc. vol. 57 (195i)

10. The boundary values of a class of meromorphic functions, Duke Math. J. vol. 19 (1952) pp. 243-252.

(14) This example, in which the unique exceptional value $w=1$ is not an asymptotic value, gives a negative answer to the question raised in p. 120 of [6]. 
11. - Les valeurs asymptotiques de quelques fonctions méromorphes dans le cercle-unitê, C.R. Acad. Sci. Paris vol. 237 (1953) pp. 16-18.

12. R. Nevanlinna, Eindeutige analytische Funktionen, Berlin, 1936.

13. M. Ohtsuka, On the cluster sets of analytic functions in a Jordan domain, J. Math. Soc. Japan vol. 2 (1950) pp. 1-15.

14. - Dirichlet problems on Riemann surfaces and conformal mappings, Nagoya Math. J. vol. 3 (1951) pp. 91-137.

15. S. Saks, Theory of the integral, Warsaw, 1937.

16. W. Seidel, On the distribution of values of bounded analytic functions, Trans. Amer. Math. Soc. vol. 36 (1934) pp. 201-226.

HARVARD UNIVERSITY,

Cambridge, Mass.

NAGOYA UNIVERSITY,

Nagoya, Japan. 